\title{
Respostas da comunidade zooplanctônica à formação do reservatório de Dona Francisca, Rio Grande do Sul, Brasil
}

\author{
Catarina da Silva Pedrozo, Fabiana Schneck, Albano Schwarzbold \& Roberto Nascimento de Farias
}

Departamento de Ecologia, Universidade Federal do Rio Grande do Sul, Av. Bento Gonçalves 9500, Caixa Postal 15007, 91501-970 Porto Alegre, RS, Brasil. (catarina.pedrozo@ufrgs.br)

\begin{abstract}
Zooplankton community responses to the impoundment of Dona Francisca reservoir, Rio Grande do Sul, Brazil. We investigated the changes in species richness, density and composition of the zooplankton community in response to the formation of a reservoir in the area of the Dona Francisca hydroelectric plant. Zooplankton samples were collected quarterly over four years, comprising two years before and two years after the formation of the reservoir. Species richness and density of Cladocera, Copepoda and Rotifera increased after the filling of the reservoir. Further, the zooplankton community showed a clear change in species composition between the periods before and after the formation of the reservoir. The distinct species composition between the two periods was related to changes in limnological characteristics due to the formation of the reservoir.
\end{abstract}

KEYWORDS. Density, richness, transition, river, reservoir.

RESUMO. Nós investigamos as mudanças na riqueza, densidade e composição da comunidade zooplanctônica em resposta à formação de um reservatório na área da usina hidrelétrica Dona Francisca. Amostras de zooplâncton foram coletadas trimestralmente durante quatro anos, compreendendo dois anos antes e dois anos após a formação do reservatório. A riqueza de espécies e a densidade de Cladocera, Copepoda e Rotifera aumentaram após o enchimento do reservatório. Além disso, a comunidade zooplanctônica apresentou uma nítida alteração na composição de espécies entre os períodos antes e após a formação do reservatório. A distinta composição de espécies entre os dois períodos esteve relacionada às alterações nas características limnológicas decorrentes da formação do reservatório.

PALAVRAS CHAVE. Densidade, riqueza, transição, rio, reservatório.

A construção de uma represa é uma atividade necessária à produção de energia, fonte de água potável, navegação, entre outros (Sternberg, 2006). Porém, resulta também em problemas sociais, ambientais e econômicos, como a perda da biodiversidade, emigrações humanas e redução pesqueira, muitos deles irreversíveis, sendo que nem todos ocorrem ao mesmo tempo (Straskraba \& Tundisi, 1999). Dentre as alterações que ocorrem devido à formação de reservatórios, podem ser citados o comportamento térmico da coluna de água, os padrões de sedimentação e circulação das massas de água, a dinâmica dos gases, a ciclagem de nutrientes e a estrutura das comunidades aquáticas (TUNDISI et al., 1993).

Represas apresentam diferenças espaciais na circulação vertical e horizontal e grande variabilidade na hidrodinâmica, que depende da morfometria dos influxos dos tributários e dos efeitos das condições climatológicas e hidrológicas (MATsumura-TundisI \& TundisI, 2005). Essas condições físicas, que têm também consequências biogeoquímicas, influenciam a distribuição e sucessão de organismos e a produtividade e biomassa das comunidades (Tundisi \& MatsumuraTuNDISI, 2008). Nota-se ainda uma mudança sequencial na dominância de espécies e comunidades de ambientes lóticos para aquelas de águas mais calmas (ACKERMAN et al., 1973). Estudos sobre a sucessão espacial e temporal das comunidades biológicas e sua relação com os processos hidrodinâmicos e ecológicos que operam dentro dos reservatórios são fundamentais para o acompanhamento do estágio de evolução destes ecossistemas (TuNDISI, 1986).
As mudanças nas condições limnológicas na transição para o ecossistema reservatório podem ser monitoradas por determinadas características da comunidade zooplanctônica, uma vez que tais organismos podem ser favorecidos pela formação de um reservatório, principalmente devido à redução do fluxo da corrente da água. Desta forma, os organismos do zooplâncton podem desenvolver grandes populações por apresentarem taxas de crescimento populacionais comumente altas em ambientes lênticos (MARzolf, 1990). De acordo com Rocha et al. (1999) esta comunidade pode estabelecer assembleias diversificadas em períodos relativamente curtos após o represamento de um rio.

Neste trabalho, avaliamos os efeitos da construção de um reservatório subtropical sobre a comunidade zooplanctônica. Nossos principais objetivos foram avaliar se a riqueza, densidade e composição da comunidade zooplanctônica diferiram entre o período anterior e o período posterior à formação do reservatório.

\section{MATERIAL E MÉTODOS}

Área de estudo e amostragem. O estudo foi realizado no empreendimento da Usina Hidrelétrica Dona Francisca (UHDF) localizada no Rio Jacuí, entre os municípios de Agudo e Dona Francisca, no estado do Rio Grande do Sul. A barragem está localizada à jusante de uma série de barragens/reservatórios em cascata: Passo Real, Salto do Jacuí e Itaúba (Fig.1). O Reservatório Dona Francisca inunda uma área de $19 \mathrm{~km}^{2}$ a uma cota de $94,5 \mathrm{~m}$, da qual $5,63 \mathrm{~km}^{2}$ 


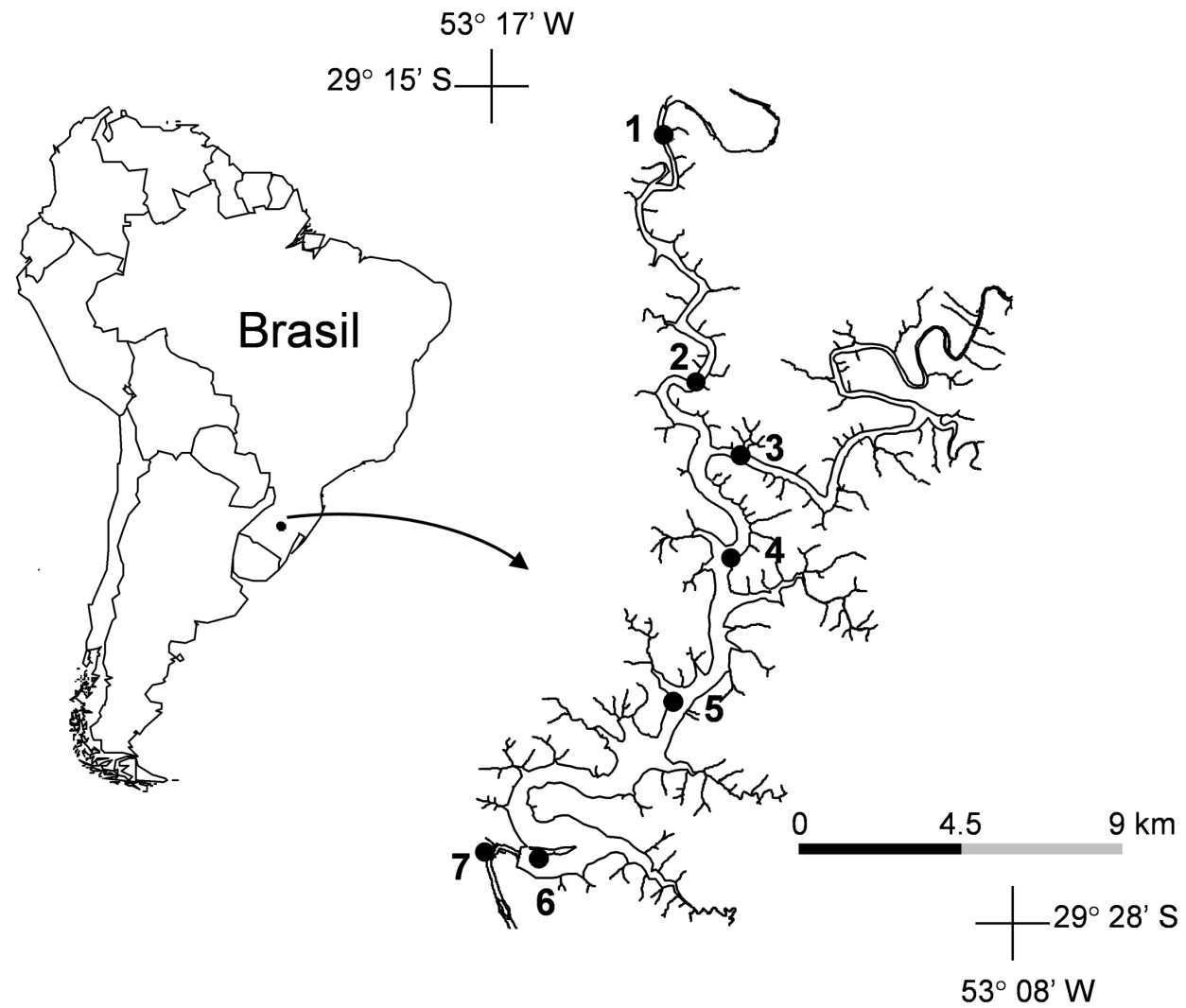

Fig.1. Localização da Usina Hidrelétrica Dona Francisca no Estado do Rio Grande do Sul, Brasil. Os sete pontos pretos correspondem aos locais de coleta.

constituíam o antigo leito do rio. A área de drenagem do reservatório é de $13.200 \mathrm{~km}^{2}$. O volume total na cota de $94,5 \mathrm{~m}$ é de $0,335 \mathrm{~km}^{3}$. O reservatório tem um baixo tempo de retenção da água, usualmente 15 dias, sendo predominantemente mesotrófico e monomítico quente (RodRigues et al., 2012).

As amostragens foram realizadas trimestralmente, em sete estações de amostragem, de outubro de 1998 a setembro de 2002, totalizando 16 trimestres e compreendendo dois anos anteriores ao enchimento do reservatório ( 8 trimestres: outubro de 1998 a setembro de 2000) e dois anos posteriores ( 8 trimestres: dezembro de 2000 a setembro de 2002). As amostragens do zooplâncton foram feitas a $0,30 \mathrm{~m}$ da superfície, no eixo principal da seção, na área afetada pela formação do reservatório. Foram coletados 300 litros de água, retirados com o auxílio de bomba de sucção e filtrados com rede de plâncton de $65 \mu \mathrm{m}$ de abertura de malha. As amostras foram preservadas com formaldeído 4\% tamponado com bórax. As variáveis físicas e químicas da água utilizadas no estudo foram: turbidez, fósforo total, nitrato, nitrogênio amoniacal, $\mathrm{pH}$, temperatura da água e condutividade elétrica (Tab. I). Coletas de água para estas análises foram realizadas concomitantemente às coletas dos organismos do zooplâncton.

Análises dos dados. As análises qualitativas do zooplâncton foram realizadas em microscópio óptico com aumento de até 400x. Os espécimes de Cladocera e Copepoda foram dissecados em glicerina com agulhas de dissecção, ao microscópio estereoscópico com aumento de até 60x. Para a identificação das espécies foram utilizadas chaves de RUTTNER-Kolisko (1974), Koste (1978), Reid (1985), Montú \& Goeden (1986), Segers (1995) e Elmoor-Loureiro (1997). As análises quantitativas foram realizadas através de contagens ao microscópio estereoscópico. Para as contagens de indivíduos pertencentes aos grupos de Cladocera e Copepoda (copepoditos e adultos) foi utilizada câmara de contagem de Bogorov. Para as contagens de Rotifera e náuplios de Copepoda foi utilizada câmara de SedgwickRafter em microscópio óptico. Os valores de densidade obtidos foram expressos em número de indivíduos por metro cúbico de água (ind. $\mathrm{m}^{-3}$ ).

A análise do índice de constância das espécies foi baseada em DAJOZ (1973). Espécies ocasionais são aquelas que ocorrem entre zero e $25 \%$ das amostras;

Tab. I. Média \pm desvio padrão das variáveis ambientais nos períodos antes e após a formação do reservatório, entre outubro de 1998 e setembro de 2002, no reservatório da Usina Hidrelétrica Dona Francisca, Rio Grande do Sul, Brasil.

\begin{tabular}{lcc}
\hline Variáveis ambientais & Antes & Após \\
\hline Temperatura da água $\left({ }^{\circ} \mathrm{C}\right)$ & $19,7 \pm 3,5$ & $21,1 \pm 3,5$ \\
Turbidez $(\mathrm{NTU})$ & $32 \pm 16$ & $22 \pm 14$ \\
Condutividade $\left(\mu \mathrm{S} . \mathrm{cm}^{-1}\right)$ & $27,9 \pm 12,8$ & $43,0 \pm 6,9$ \\
$\mathrm{pH}$ & $7,0 \pm 0,5$ & $6,8 \pm 0,4$ \\
Fósforo total $\left(\mu \mathrm{g} . \mathrm{L}^{-1}\right)$ & $54,50 \pm 45,25$ & $51,1 \pm 32,64$ \\
Nitrato $\left(\mathrm{mg} \cdot \mathrm{L}^{-1}\right)$ & $0,16 \pm 0,31$ & $0,33 \pm 0,40$ \\
Nitrogênio amoniacal $\left(\mathrm{mg} \cdot \mathrm{L}^{-1}\right)$ & $0,35 \pm 0,32$ & $0,48 \pm 0,46$ \\
\hline
\end{tabular}


aquelas que ocorrem em $>25 \%$ são consideradas acessórias e $>50 \%$ são classificadas como constantes.

As espécies associadas a cada período foram identificadas através da Análise de Espécies Indicadoras (Dufrêne \& Legendre, 1997). A análise foi aplicada sobre a matriz de densidade, com os períodos antes e após a formação do reservatório como variáveis de agrupamento. Os valores indicativos foram testados através do teste de Monte Carlo (999 permutações).

Para avaliar as relações entre a distribuição das espécies indicadoras de cada período (Análise de Espécies Indicadoras) e os dados ambientais foi aplicada uma Análise de Correspondência Canônica (CCA) (TER BRAAK, 1986). A matriz biótica sofreu transformação $\log ($ densidade +1$)$ e a matriz de variáveis ambientais foi padronizada pela média e desvio padrão. A probabilidade dos autovalores dos eixos de ordenação terem sido atribuídos ao acaso foi avaliada através de teste de Monte Carlo (999 permutações). A Análise de Espécies Indicadoras e a CCA foram realizadas no software PCOrd (MCCune \& MEFFord, 1999).

\section{RESULTADOS}

Comunidade zooplanctônica. $\mathrm{O}$ zooplâncton foi representado por Cladocera, Copepoda e Rotifera, estando este último grupo presente em todas as estações amostrais ao longo de todo o período do estudo, constituindo sempre as maiores densidades. Foram encontrados 77 táxons de organismos zooplanctônicos. Os rotíferos foram representados por 57 táxons, os cladóceros por 13 e os copépodos por 7. Os rotíferos identificados pertencema 18 famílias, sendo Brachionidae a mais representativa (18 espécies), destacando-se ainda Lecanidae (6 espécies) e Trichocercidae (5 espécies). Os cladóceros identificados pertencem a seis famílias e os copépodos foram representados por apenas duas famílias. Do total de 77 táxons, 58 foram registrados no período anterior à formação do reservatório e 68 no período após a formação do reservatório.

Quatorze táxons foram exclusivos do período anterior à formação do reservatório e 24 exclusivos do período posterior à formação (Tab. II). Todas as espécies exclusivas em cada período apresentaram baixas frequências de ocorrência em todo o sistema, sendo consideradas ocasionais. As espécies constantes em ambos os períodos foram os cladóceros Bosmina hagmani Stingelin, 1904 e Bosminopsis deitersi Stingelin, 1904, e os rotíferos Kellicottia longispina (Kellicot, 1879), K. cochlearis (Gosse, 1851), Polyarthra sp. e Ploesoma truncatum (Levander, 1894). Já as espécies constantes apenas no período anterior à formação do reservatório foram Collotheca $\mathrm{sp}$. (entre os rotíferos) e Bosmina longirostris O. F. Müller, 1785 (entre os cladóceros). Os rotíferos Keratella americana hispida Lauterborn, 1898, Filinia longiseta (Ehrenberg, 1834), Trichocerca capucina (Wierzejski \& Zacharias,
Tab. II. Composição da comunidade zooplanctônica e sua frequência de ocorrência (\%) nos períodos antes e após a formação do reservatório, entre outubro de 1998 e setembro de 2002 no reservatório da Usina Hidrelétrica Dona Francisca, Rio Grande do Sul, Brasil.

\begin{tabular}{|c|c|c|}
\hline & Antes & Após \\
\hline \multicolumn{3}{|l|}{ ROTIFERA } \\
\hline \multicolumn{3}{|l|}{ Asplanchnidae } \\
\hline Asplanchna sp. & 0 & 5 \\
\hline Bdelloidea & 0 & 16 \\
\hline \multicolumn{3}{|l|}{ Brachionidae } \\
\hline Anuraeopsis fissa Gosse, 1851 & 0 & 11 \\
\hline Brachionus ahlstromi Lindeman, 1939 & 9 & 16 \\
\hline Brachionus angularis orientalis Sudzuki, 1989 & 2 & 7 \\
\hline Brachionus calyciflorus Pallas, 1766 & 2 & 12 \\
\hline Brachionus caudatus Barrois \& Daday, 1894 & 2 & 4 \\
\hline Brachionus dolabratus Harring, 1914 & 4 & 9 \\
\hline Brachionus falcatus Zacharias, 1898 & 0 & 2 \\
\hline Brachionus mirus Daday, 1905 & 2 & 0 \\
\hline Brachionus quadridentatus Hermann, 1783 & 2 & 0 \\
\hline Kellicottia longispina (Kellicot, 1879) & 80 & 82 \\
\hline Keratella americana Carlin, 1943 & 73 & 45 \\
\hline Keratella americana hispida Lauterborn, 1898 & 0 & 55 \\
\hline Keratella cochlearis (Gosse, 1851) & 85 & 91 \\
\hline Keratella lenzi Hauer, 1953 & 14 & 7 \\
\hline Keratella tecta (Gosse, 1851) & 30 & 12 \\
\hline Keratella tropica (Apstein, 1907) & 44 & 7 \\
\hline Plationus patulus patulus (Müller, 1786) & 4 & 0 \\
\hline Platyias quadricornis (Ehrenberg, 1832) & 2 & 18 \\
\hline \multicolumn{3}{|l|}{ Collothecidae } \\
\hline Collotheca trilobata (Collins, 1872) & 2 & 9 \\
\hline Collotheca sp. & 60 & 20 \\
\hline \multicolumn{3}{|l|}{ Conochilidae } \\
\hline Conochilus (Conochiloides) coenobasis (Skorikov, 1914) & 20 & 4 \\
\hline Conochilus (Conochiloides) dossuarius Hudson, 1885 & 2 & 0 \\
\hline Conochilus (Conochilus) unicornis Rousselet, 1892 & 41 & 12 \\
\hline \multicolumn{3}{|l|}{ Euchlanidae } \\
\hline Euchlanis dilatata Ehrenberg, 1832 & 4 & 14 \\
\hline \multicolumn{3}{|l|}{ Filinidae } \\
\hline Filinia longiseta (Ehrenberg, 1834) & 23 & 54 \\
\hline Filinia opoliensis (Zacharias, 1898) & 0 & 2 \\
\hline \multicolumn{3}{|l|}{ Gastropodidae } \\
\hline Ascomorpha ecaudis Perty, 1850 & 0 & 11 \\
\hline Gastropus stylifer (Imhof, 1891) & 0 & 6 \\
\hline Gastropus sp. & 0 & 9 \\
\hline \multicolumn{3}{|l|}{ Hexarthridae } \\
\hline Hexarthra sp. & 2 & 30 \\
\hline \multicolumn{3}{|l|}{ Lecanidae } \\
\hline Lecane bulla (Gosse, 1851$)$ & 14 & 13 \\
\hline Lecane luna (Müller, 1776) & 9 & 4 \\
\hline Lecane lunaris (Ehrenberg, 1832) & 11 & 14 \\
\hline Lecane quadridentata (Ehrenberg, 1830) & 2 & 0 \\
\hline Lecane stenroosi (Meissner, 1908) & 2 & 2 \\
\hline Lecane sp. Nitzsch, 1827 & 9 & 7 \\
\hline \multicolumn{3}{|l|}{ Lepadellidae } \\
\hline Lepadella (Lepadella) patella (Müller, 1773) & 18 & 9 \\
\hline Lepadella sp. & 2 & 2 \\
\hline \multicolumn{3}{|l|}{ Mytilinidae } \\
\hline Lophocharis oxysternon (Gosse, 1851) & 0 & 2 \\
\hline Mytilina sp. & 2 & 0 \\
\hline \multicolumn{3}{|l|}{ Notommatidae } \\
\hline Cephalodella gibba (Ehrenberg, 1830) & 5 & 2 \\
\hline
\end{tabular}


Tab. II. (Cont.)

\begin{tabular}{|c|c|c|}
\hline & Antes & Após \\
\hline \multicolumn{3}{|l|}{ Notommatidae } \\
\hline Notommata sp. & 4 & 0 \\
\hline \multicolumn{3}{|l|}{ Philodinidae } \\
\hline Philodina sp. & 2 & 5 \\
\hline \multicolumn{3}{|l|}{ Synchaetidae } \\
\hline Ploesoma truncatum (Levander, 1894) & 73 & 66 \\
\hline Polyarthra sp. & 87 & 75 \\
\hline Synchaeta oblonga Ehrenberg, 1832 & 0 & 2 \\
\hline Synchaeta sp. & 36 & 2 \\
\hline \multicolumn{3}{|l|}{ Testudinellidae } \\
\hline Pompholyx complanata Gosse, 1851 & 25 & 16 \\
\hline Pompholyx sulcata Hudson, 1885 & 0 & 7 \\
\hline \multicolumn{3}{|l|}{ Trichocercidae } \\
\hline $\begin{array}{l}\text { Trichocerca capucina (Wierzejski \& Zacharias, } \\
\text { 1893) }\end{array}$ & 18 & 59 \\
\hline Trichocerca cylindrica (Imhof, 1891) & 2 & 11 \\
\hline Trichocerca multicrinis (Kellicott, 1897) & 14 & 0 \\
\hline Trichocerca similis (Wierzejski, 1893) & 36 & 18 \\
\hline Trichocerca sp. & 41 & 43 \\
\hline \multicolumn{3}{|l|}{ Trichotriidae } \\
\hline Macrochaetus sp. & 2 & 0 \\
\hline Trichotria tetractis (Ehrenberg, 1830) & 0 & 5 \\
\hline \multicolumn{3}{|l|}{ CLADOCERA } \\
\hline \multicolumn{3}{|l|}{ Bosminidae } \\
\hline Bosmina longirostris (O. F. Müller, 1785) & 71 & 34 \\
\hline Bosmina hagmani Stingelin, 1904 & 52 & 61 \\
\hline Bosminopsis deitersi Richard, 1895 & 61 & 93 \\
\hline \multicolumn{3}{|l|}{ Daphnidae } \\
\hline Ceriodaphnia cornuta Sars, 1886 & 10 & 16 \\
\hline Ceriodaphnia sp. & 0 & 7 \\
\hline Daphnia sp. & 2 & 0 \\
\hline \multicolumn{3}{|l|}{ Sididae } \\
\hline Diaphanosoma birgei Korinek, 1981 & 2 & 5 \\
\hline Diaphanosoma fluviatile Hansen, 1899 & 5 & 2 \\
\hline Diaphanosoma sp. & 4 & 7 \\
\hline \multicolumn{3}{|l|}{ Ilyocryptidae } \\
\hline Ilyocryptus spinifer Herrick, 1884 & 0 & 2 \\
\hline \multicolumn{3}{|l|}{ Macrothricidae } \\
\hline Macrothrix sp. & 2 & 4 \\
\hline \multicolumn{3}{|l|}{ Moinidae } \\
\hline Moina micrura Kurz, 1874 & 2 & 13 \\
\hline Moina minuta Hansen, 1899 & 16 & 25 \\
\hline \multicolumn{3}{|l|}{ COPEPODA } \\
\hline \multicolumn{3}{|l|}{ Cyclopidae } \\
\hline \multicolumn{3}{|l|}{ Cyclopinae } \\
\hline Acanthocyclops vernalis (Fischer, 1853) & 0 & 4 \\
\hline Eucyclops sp. & 0 & 2 \\
\hline Mesocyclops longisetus (Thiébaud, 1914) & 0 & 2 \\
\hline Metacyclops sp. & 0 & 11 \\
\hline Microcyclops anceps (Richard, 1897) & 0 & 2 \\
\hline Thermocyclops minutus (Lowndes, 1934) & 13 & 57 \\
\hline \multicolumn{3}{|l|}{ Diaptomidae } \\
\hline Notodiaptomus incompositus Brian, 1926 & 7 & 5 \\
\hline
\end{tabular}

1893) e o copépodo Thermocyclops minutus Lowndes, 1934 foram constantes no período após a formação do reservatório.

Houve uma tendência de aumento na riqueza de espécies e na densidade de organismos em todos os pontos de coleta imediatamente após o enchimento do reservatório (Figs. 2-9). Esta tendência foi observada tanto para a comunidade como para cada um dos grupos zooplanctônicos, destacando-se o aumento mais pronunciado na densidade de Rotifera. Esse período de aumento na riqueza e densidade em decorrência da formação do reservatório foi seguido por uma grande variabilidade temporal nestes atributos ao longo dos trimestres seguintes em todas as estações de amostragem, porém sempre com uma tendência de maior densidade quando comparado ao período anterior à formação do reservatório.

A Análise de Espécies Indicadoras $(\alpha=0,05)$ indicou 22 táxons, sendo que somente dois foram significativamente correlacionadas à época anterior ao enchimento do reservatório: os rotíferos Keratella tropica (Apstein, 1907) e Trichocerca multicrinis (Kellicott, 1897). Já os táxons indicadores do período após a formação do reservatório foram os cladóceros Bosmina hagmani, Bosminopsis deitersi, Moina micrura Kurz, 1874, Moina minuta Hansen, 1899; os copépodos Metacyclops sp., Thermocyclops minutus, Copepoditos Cyclopoida; os rotíferos Anuraeopsis fissa Gosse, 1851, Ascomorpha ecaudis Perty, 1850, Bdelloidea, Brachionus calyciflorus Pallas, 1766, Euchlanis dilatata Ehrenberg, 1832, Filinia longiseta, Gastropus sp., Hexarthra sp., Keratella americana hispida, Keratella cochlearis Gosse, 1851, Platyias quadricornis (Ehrenberg, 1832), Polyarthra sp. e Trichocerca capucina.

Relação entre a comunidade zooplanctônica e as variáveis ambientais. Os dois primeiros eixos da Análise de Correspondência Canônica (CCA) indicam uma nítida separação das comunidades antes e após a formação do reservatório, demonstrando a ocorrência de diferenças limnológicas e biológicas entre os períodos (Figs. 10-11). Os dois primeiros eixos da CCA explicaram $13,8 \%$ da variância total (eixo $1=8,6 \%$; eixo $2=5,2 \%$; significância de ambos os eixos: $P=$ 0,001). As correlações espécie-ambiente foram altas para os dois eixos $(\mathrm{r}=0,781$ e 0,837 , respectivamente; $P=0,001$ em ambos os casos), indicando uma forte relação entre as variáveis ambientais e a distribuição das espécies.

Condutividade elétrica apresentou correlação positiva com o primeiro eixo $(\mathrm{r}=0,832)$, e o $\mathrm{pH}$, negativa $(\mathrm{r}=-0,587)$. No segundo eixo, o maior peso para o ordenamento foi atribuído à turbidez $(\mathrm{r}=-0,807)$, negativamente correlacionada com o eixo, e à temperatura da água $(\mathrm{r}=0,770)$, fósforo total $(r=0,308)$ e nitrogênio amoniacal $(r=0,360)$, positivamente direcionados sobre o eixo. Sobre o lado negativo do primeiro eixo estão localizadas Trichocerca multicrinis e Keratella tropica, enquanto que na esfera positiva estão espécies indicadoras do período após a formação do reservatório, como Ascomorpha ecaudis, Hexarthra sp., Metacyclops sp., Anuraeopsis fissa, Euchlanis dilatata e Keratella americana hispida. 


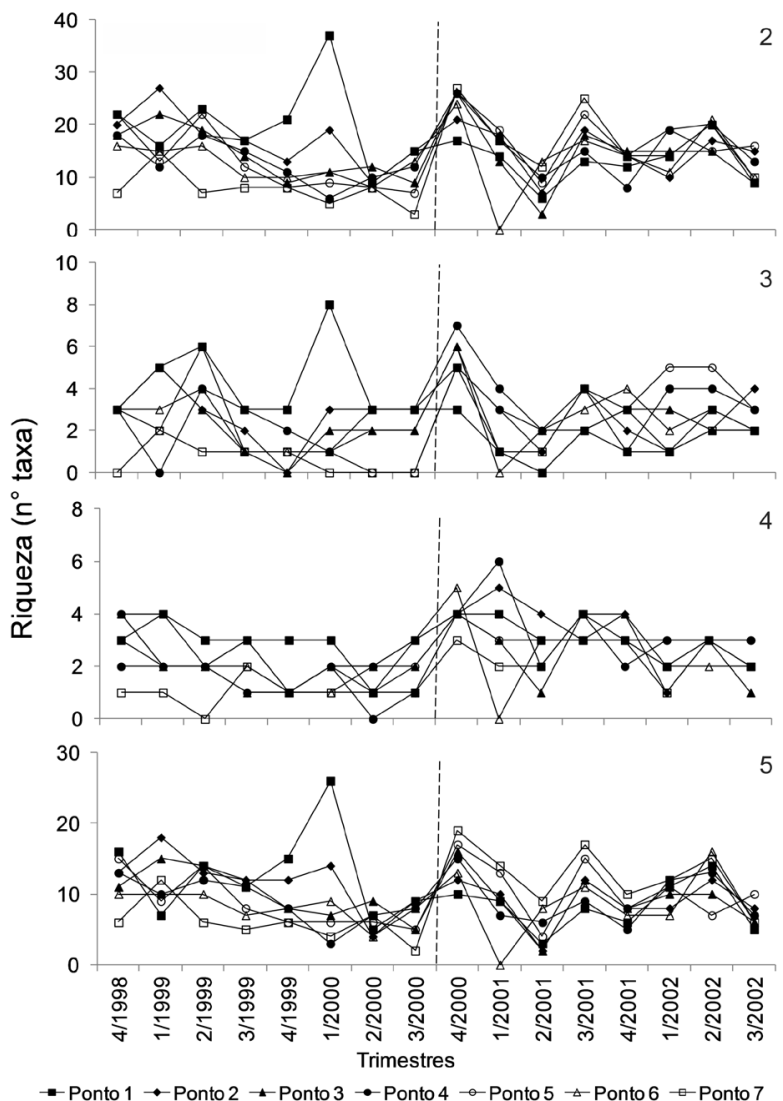

Figs 2-5. Riqueza de táxons zooplanctônicos ao longo de 16 coletas trimestrais entre outubro de 1998 e setembro de 2002 no reservatório da Usina Hidrelétrica Dona Francisca, Rio Grande do Sul, Brasil para: 2, comunidade total; 3, Cladocera; 4, Copepoda e 5, Rotifera. A linha vertical delimita a data do enchimento do reservatório.

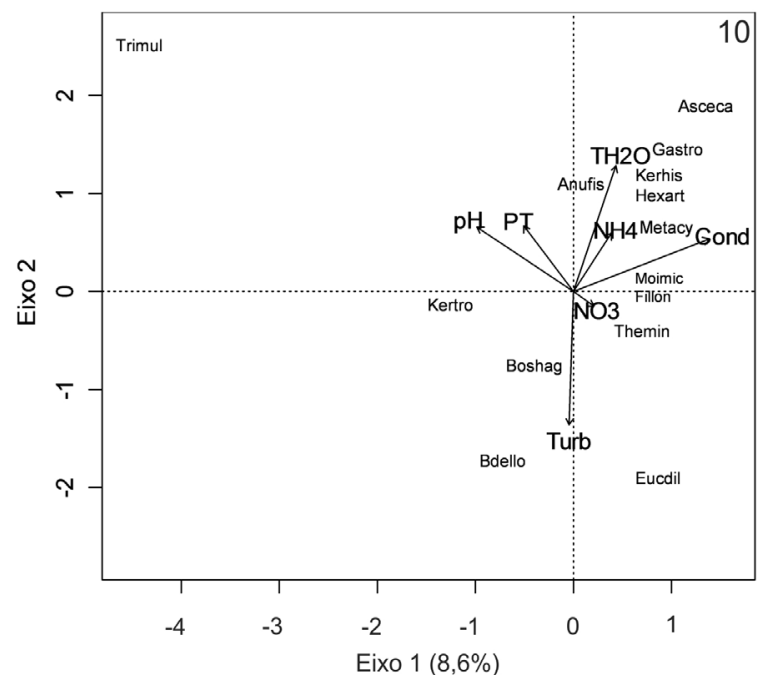

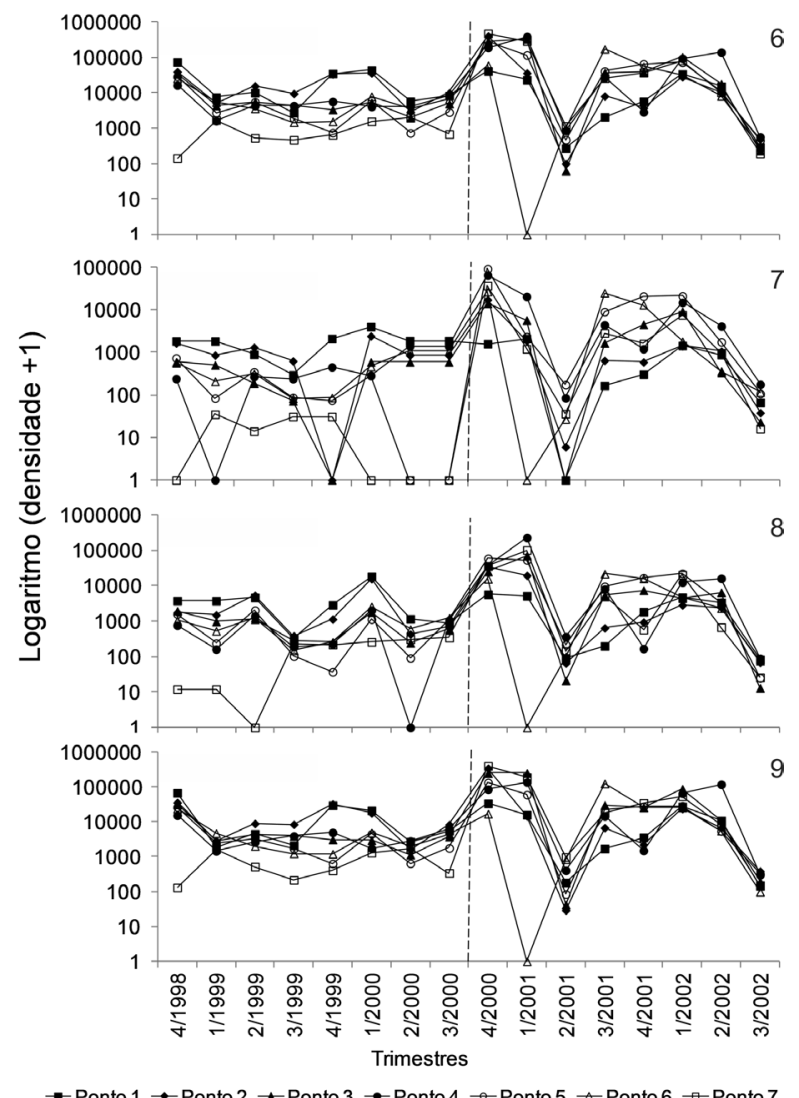

$\rightarrow$ Ponto $1 \rightarrow$ Ponto $2 \rightarrow$-Ponto $3 \rightarrow$-Ponto $4 \rightarrow$-Ponto $5 \rightarrow$ Ponto 6 -Ponto 7

Figs 6-9. Densidade $(\log [\mathrm{x}+1])$ de táxons zooplanctônicos ao longo de 16 coletas trimestrais entre outubro de 1998 e setembro de 2002 no reservatório da Usina Hidrelétrica Dona Francisca, Rio Grande do Sul, Brasil para: 6, comunidade total; 7, Cladocera; 8, Copepoda e 9, Rotifera. A linha vertical delimita a data do enchimento do reservatório.

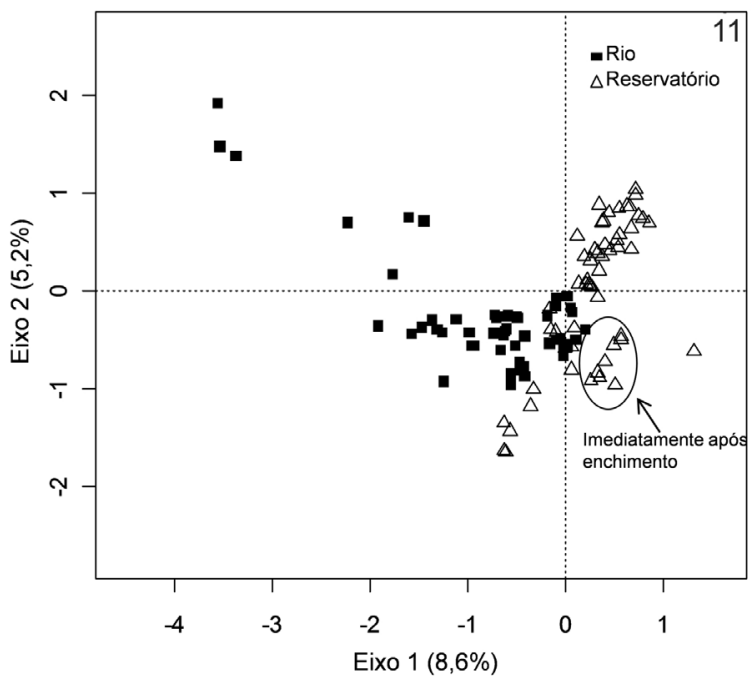

Figs 10,11. Diagramas de dispersão obtido a partir da Análise de Correspondência Canônica (CCA) aplicada sobre as espécies indicadoras (10) e as variáveis ambientais (11) (Anufis, Anuraeopsis fissa; Asceca, Ascomorpha ecaudis; Bdello, Bdelloidea; Boshag, Bosmina hagmani; Eucdil, Euchlanis dilatata; Fillon, Filinia longiseta; Gastro, Gastropus sp.; Hexart, Hexarthra spp.; Kerhis, Keratella americana hispida; Kertro, Keratella tropica; Metacy, Metacyclops sp.; Moimic, Moina micrura; Themin, Thermocyclops minutus; Trimul, Trichocerca multicrinis; Cond, condutividade elétrica; NH4, nitrogênio amoniacal; NO3, nitrato; pH, potencial hidrogeniônico; PT, fósforo total; TH2O, temperatura da água; Turb, turbidez).

\section{DISCUSSÃO}

Comunidade zooplanctônica. Entre as espécies zooplanctônicas observadas na fase anterior ao enchimento do reservatório, quando o sistema apresentava características lóticas, muitas são características de habitat litorâneo ou perifítico e algumas raramente são encontradas no plâncton, como Notommata sp. e Mytilina sp. Outro exemplo é a maior ocorrência de espécies de Lecane e Lepadella nesta fase. Estes rotíferos loricados 
predominam em ambientes lóticos devido à habilidade de crescer mesmo em condições de rápido fluxo de água (PICARD \& Lair, 2003).

Foi observada uma tendência de maior riqueza imediatamente após o enchimento do reservatório. Esta maior riqueza pode estar relacionada ao aumento na densidade de organismos no mesmo período, uma vez que o número de indivíduos amostrados e a riqueza de espécies em uma amostra são positivamente correlacionados (McCABe \& Gotelli, 2000). Além disso, a comunidade zooplanctônica pode apresentar-se mais diversificada neste período, uma vez que as novas características físicas do ambiente, como a redução da velocidade da água, podem favorecer este grupo (Rocha et al., 1999). Entre as espécies registradas imediatamente após a formação do reservatório, algumas são não-planctônicas ou semi-planctônicas, como Ilyocryptus spinifer Herrick, 1884, e outras são tipicamente planctônicas, como Filinia opoliensis (Zacharias, 1898), Gastropus sp. e Synchaeta oblonga Ehrenberg, 1831. Espécies não- planctônicas podem ser facilmente arrastadas pela correnteza dos ambientes lóticos, contribuindo com a maior riqueza específica nos primeiros estágios do enchimento de um reservatório. Resultados semelhantes foram observados por TAKAHASHI et al. (2008) durante o enchimento do Reservatório de Santa Clara, no Paraná, salientando a contribuição expressiva de espécies nãoplanctônicas no início do enchimento do reservatório, sendo que esta contribuição diminui com a formação do reservatório e com o consequente aumento da ocorrência de espécies planctônicas. Em qualquer rio a quantidade de organismos do plâncton aumenta em direção à foz, portanto, quanto maior o tempo de residência da água no barramento de um rio, maior é a chance de que espécies de hábito planctônico tenham mais condições de se reproduzirem, uma vez que estes organismos apresentam altas taxas de crescimento populacional em ambientes lênticos (Marzolf, 1990).

A colonização de um ambiente recém-formado, como um reservatório, depende da biota presente nos ambientes ribeirinhos e lacustres da bacia de drenagem (Agostinho et al., 1999). Em um reservatório, esta colonização ocorre durante o período dominado por condições heterotróficas induzidas pelo fluxo e pela subsequente decomposição da matéria orgânica terrestre (Margalef, 1983). Detalhes dos eventos que seguem este período inicial heterotrófico são imprevisíveis, assim como as respostas das comunidades às subsequentes perturbações, naturais ou manipuladas. Neste estudo, a alteração na densidade de organismos, com um aumento após a formação do reservatório, reflete estas perturbações. Um grande aumento na densidade do zooplâncton, principalmente de rotíferos, na fase de reservatório, também foi registrado nesta fase por PINELAlloul et al. (1989), CAmpbell et al. (1998), Garrido \& Bozelli (2000) e TAKAhAshi et al. (2008), como resultante das mudanças na quantidade e na qualidade dos recursos alimentares. Além disso, a comunidade zooplanctônica pode ter sido favorecida pela diminuição da turbulência e do fluxo da água, acarretando em um aumento na densidade de organismos em função da possível ocorrência de condições mais adequadas à reprodução e à sobrevivência no novo ambiente formado.

Relação entre a comunidade zooplanctônica e as variáveis ambientais. A baixa explicação encontrada na Análise de Correspondência Canônica (13,8\% nos dois primeiros eixos) é esperada em análises de dados ecológicos devido à complexidade dos fatores atuantes na determinação da estrutura das comunidades (TER BraAk \& Prentice, 1988). Este resultado pode ainda estar relacionado ao fato de que as variáveis ambientais utilizadas na CCA não refletem diretamente as alterações físicas mais abruptas no ambiente após a formação do reservatório, como alterações na velocidade da corrente, tempo de retenção da água e profundidade da coluna d'água. Porém, a baixa explicação encontrada não comprometeu as análises das relações espécie-ambiente, uma vez que as correlações espécie-ambiente foram altas e significativas para os dois eixos, indicando uma estreita relação entre as variáveis ambientais avaliadas e a distribuição das espécies e uma nítida diferenciação das comunidades de acordo com o período, resultando em comunidades distintas à medida que o ecossistema passa da condição de rio para a condição de reservatório. De forma semelhante, Matsumura-Tundisi et al. (1991) registraram que a composição específica do zooplâncton apresentou mudanças nítidas e rápidas durante a fase de enchimento de um reservatório em Tocantins, Brasil.

As alterações observadas na composição da comunidade podem ser atribuídas à provável substituição de espécies adaptadas a ambientes lóticos por outras melhor adaptadas ao novo ambiente criado pela formação do reservatório (e.g., TAKAHASHI et al., 2008). Além disso, as mudanças ocorridas nas variáveis limnológicas decorrentes do represamento, como diminuição na turbidez, aumento na temperatura da água e na concentração de nutrientes, podem causar uma rápida alteração na composição de espécies e um aumento na biomassa zooplanctônica (MARZOLF, 1990).

Os maiores valores de turbidez da água no período anterior à formação do reservatório, como evidenciado na CCA, estão relacionados ao aporte de sedimentos dos tributários do sistema e à maior turbulência da água no ambiente originalmente lótico. Neste período, apenas Keratella tropica e Trichocerca capuccina multicrinia foram consideradas indicadoras; esta última é raramente encontrada em águas abertas, sendo geralmente esporádica e pouco numerosa nestes ambientes.

Após a formação do reservatório, com o aumento do tempo de residência da água, houve diminuição da turbidez e aumento da temperatura da água, provavelmente influenciando a densidade e composição da comunidade zooplanctônica. Essa influência pode ocorrer tanto indiretamente, através do 
aumento na produção fitoplanctônica e consequente aumento na disponibilidade de recursos alimentares para o zooplâncton, como diretamente, através da ocorrência de espécies adaptadas às novas condições. Por exemplo, nesse período a representatividade de formas planctônicas foi muito maior que no período anterior à formação do reservatório, resultando em uma riqueza de 68 táxons, o que pode ser atribuído à estabilidade hídrica do sistema no novo ambiente lêntico originado com a formação do reservatório. Além disso, a correlação positiva de condutividade elétrica e nitrogênio amoniacal com o período após o enchimento está relacionada ao maior aporte de matéria orgânica e decomposição da vegetação submersa, além da possível entrada e maior concentração de efluentes orgânicos dos arroios da região no reservatório.

O número de táxons indicadores do período após a formação do reservatório foi maior que no período anterior e representado principalmente por espécies de Rotifera. Os rotíferos são organismos oportunistas e com ciclo de vida curto (r-estrategistas), adaptando-se rapidamente a alterações ambientais (AllaN, 1976), como por exemplo, as alterações nas características físicas e químicas decorrentes da formação do reservatório. Entre os rotíferos indicadores deste período estão: Anuraeopsis fissa, Keratella americana hispida, Bdelloidea, Brachionus calyciflorus, Ascomorpha ecaudis e Gastropus sp., que são descritos como predominantes em lagos tropicais e ambientes com fluxo lento, ricos em matéria orgânica. Membros da ordem Bdelloidea geralmente são encontrados no sedimento e muito ocasionalmente aparecem no plâncton. Anuraeopsis fissa é um rotífero comum em regiões tropicais e temperadas (SARMA et al., 2007) e se alimenta principalmente, ou exclusivamente, de detritos. Já Ascomorpha ecaudis é estenófaga, planctônica, cosmopolita e perene, com dieta mais ou menos especializada (POURRIOT, 1977), e B. calyciflorus é considerada por PedROzo \& RochA (2005) como tolerante à poluição orgânica.

A ocorrência de organismos de Cladocera e Copepoda como indicadores do período após a formação do reservatório pode ser justificada pelo fato de que os microcrustáceos são geralmente mais diversos em ambientes lênticos e, raramente numerosos em ecossistemas abertos como os rios (HyNES, 1970). Além disso, a presença de sólidos em suspensão nos ambientes turbulentos dos rios pode prejudicar a alimentação desses pequenos microcrustáceos, uma vez que os mesmos se alimentam em parte de materiais presos por suas patas, podendo vir a ingerir partículas de sedimento que os tornarão mais pesados e impossibilitados de se manterem na coluna d'água. Já em águas mais calmas, os microcrustáceos se estabelecem mais facilmente (Kirk \& Gilbert, 1990).

Nossos resultados indicam que o barramento do rio Jacuí para a construção da hidrelétrica de Dona Francisca alterou fortemente a comunidade zooplanctônica. A transformação de um sistema lótico em um sistema lêntico favoreceu os organismos zooplanctônicos, tendo sido observadas modificações na riqueza, densidade e composição da comunidade após a formação do reservatório.

Agradecimentos. Os autores agradecem à Dona Francisca Eletricidade (DFESA) pela disponibilização dos dados. F. Schneck foi bolsista de doutorado da Coordenação de Aperfeiçoamento de Pessoal de Nível Superior (CAPES) durante a realização deste trabalho.

\section{REFERÊNCIAS BIBLIOGRÁFICAS}

Ackerman, W. C.; White, G. F. \& Worthington, E. B. 1973. Manmade lakes: their problems and environmental effects Washington, D.C., American Geophysical Union. 847p.

Agostinho, A. A.; Miranda, L. E.; Bini, L. M.; Gomes, L. C. Thomaz, S. M. \& Suzuki, H. I. 1999. Patterns of Colonization in Neotropical Reservoirs, and Prognoses on Aging. In: Tundisi, J. G. \& Straskraba, M. eds. Theoretical Reservoir Ecology and its Applications. São Carlos, Academia Brasileira de Ciências \& Backhuys Publishers. p. 227-265.

Allan, J. D. 1976. Life history parameters in zooplankton. American Naturalist 110:165-180

Campbell, C.; Knoechel, R. \& Copeman, D. 1998. Evaluation of factors related to increased zooplankton biomass and altered species composition following impoundment of a Newfoundland reservoir. Canadian Journal of Fisheries and Aquatic Sciences 55:230-238.

Dajoz, R. 1973. Ecologia Geral. São Paulo, Vozes, Edusp. 472p.

Dufrêne, M. \& Legendre, P. 1997. Species assemblages and indicator species: the need for a flexible asymmetrical approach. Ecological Monographs 67:345-366.

Elmoor-Loureiro, L. M. A. 1997. Manual de Identificação de Cladóceros Límnicos do Brasil. Brasília, Universa. 155p.

Garrido, A. V. \& Bozelli, R. L. 2000. The study of zooplankton during the filling of the Serra da Mesa Reservoir, Tocantins River (GO, Brazil). Verhandlungen der Internationalen Vereinigung für Theoretische und Angewandte Limnologie 27:2875-2878.

HynEs, H. B. N. 1970. The ecology of running waters. Liverpool, Liverpool University Press. 555p.

KiRK, K. L. \& Gilbert, J. J. 1990. Suspended clay and the population dynamics of plankton rotifers and cladocerans. Ecology 71:17411766.

Koste, W. 1978. Rotatoria; Die Rädertiere Mitteleuropas Ein Bestimmungswerk Begründet von Max Voigt. Uberordnung monogononta, 2. Aul. Berlin, Gebrüber Borntraeger. 637p.

Margalef, R. 1983. Limnología. Barcelona, Omega. 1010p.

Marzolf, G. R. 1990. Reservoirs as environments for zooplankton. In: Thornton, K.; Kimmel, B. L. \& Payne, F. E. eds. Reservoir limnology: ecological perspectives. New York, Wiley Interscience Publication. v.7, p. 195-208.

Matsumura-Tundisi, T. \& Tundisi, J. G. 2005. Plankton richness in a eutrophic reservoir (Barra Bonita Reservoir, SP, Brazil ). Hydrobiologia 542:367-378.

Matsumura-Tundisi, T.; Tundisi, J. G.; Saggio, A.; Oliveira Neto, A. \& Espindola, E. G. 1991. Limnology of Samuel Reservoir (Brazil, Rondônia) in the Filling Phase. Verhandlungen des Internationales Verein Limnologie 24:1482-1488.

McCABE, D. J. \& Gotelli, N. J. 2000. Effects of disturbance frequency, intensity, and area on assemblages of stream macroinvertebrates. Oecologia 124:270-279.

McCune, B. \& MefFord, M. J. 1999. PC-ORD. Multivariate Analysis of Ecological Data, Version 4.10. MjM Software Design, Oregon. 237p. Montú, M. \& Goeden, I. M. 1986. Atlas dos Cladocera e Copepoda (Crustacea) do estuário da Lagoa dos Patos (Rio Grande, Brasil). Nerítica 1(2):1-134

Pedrozo, C. DA S. \& Rocha, O. 2005. Zooplankton and water quality of lakes of the Northern Coast of Rio Grande do Sul State, Brazil. Acta Limnologica Brasiliensia 17:445-464.

PicARD, V. \& LAIR, N. 2003. Laboratory approach of the growth of rotifers sampled in the Middle Loire (France) under turbulence. Journal de Recherche Oceanographique 28:196-199. 
Pinel-Alloul, B.; Mèthod, G. \& Florescu, M. 1989. Zooplankton species dynamics during impoundment and stabilization in subartic reservoir. Archiv für Hydrobiologie-Beiheft Ergebnisse der Limnologie 33:521-537.

Pourriot, R. 1977. Food and feeding habits of Rotifera. Archiv für Hydrobiologie -Beiheft Ergebnisse der Limnologie 8:243-260.

REID, J. W. 1985. Chave para identificação e lista de referências bibliográficas para espécies continentais sulamericanas de vida livre da Ordem Cyclopoida (Crustacea, Copepoda). Boletim de Zoologia 9:17-143.

Rocha, O.; Tundisi, T. M.; Espindola, E. L. G.; Roche, K. F. \& Rietzler, A. C. 1999. Ecological theory applied to reservoir zooplankton. In: Tundisi, J. G. \& Straskraba, M. eds. Theoretical Reservoir Ecology and its Applications. São Carlos, Academy of Science and Backhuys Publishers. p. 457-476.

Rodrigues, L. M.; Schwarzbold, A. \& Oliveira, M. A. 2012. Spatial and temporal variation of Dona Francisca reservoir (Jacuí River, RS), a subtropical reservoir. Acta Scientiarum, Biological Sciences (in press).

Ruttner-Kolisko, A. 1974. Plankton Rotifers: Biology and Taxonomy. Stuttgart, Gebrüder Ranz.146p.

Sarma, S. S. S.; Rivera, S. A.; Hinojosa, F. E. \& Nandini, S. 2007. Combined influence of food level and inoculation density on competition between Anuraeopsis fissa and Brachionus patulus or Brachionus macracanthus (Rotifera: Brachionidae). Russian Journal of Ecology 38(5):381-390.

Segers, H. 1995. Rotifera Volume 2: The Lecanidae (Monogononta). The Hague, SPB Academic Publishing. 226p.
Sternberg, R. 2006. Damming the river: a changing perspective on altering nature. Renewable and Sustainable Energy Reviews 10:165-197.

Straskraba, M. \& Tundisi, J. G. 1999. Reservoir Ecosystem Functioning: Theory and Application. In: Tundisi, J. G. \& Straskraba, M. eds. Theoretical Reservoir Ecology and its Applications. São Carlos, Academy of Science and Backhuys Publishers. p. 565-583.

Takahashi, E. M.; Lansac-Tôha, F. A,; Dias, J. D.; Bonecker, C. C. \& Velho, L. F. M. 2008. Spatial variations in the zooplankton community from the Corumbá Reservoir, Goiás State, in distinct hydrological periods. Acta Scientiarum, Biological Sciences 31(3):227-234

Ter BraAK, C. J. F. 1986. Canonical Correspondence Analysis: a new eigenvector technique for multivariate direct gradient analysis. Ecology 67:1167-1179.

Ter Braak, C. J. F. \& Prentice, I. C. 1988. The theory of gradient analysis. Advances in Ecological Research 18:271-317.

Tundisi, J. G. 1986. Limnologia de Represas Artificiais. Boletim de Hidráulica e Saneamento EESC/USP 11:1-46.

Tundisi, J. G. \& Matsumura-Tundisi, T. 2008. Limnologia. São Paulo, Oficina de Textos. 632p.

Tundisi, J. G.; Matsumura-Tundisi, T. \& Calijuri, M. C. 1993 Limnology and management of reservoirs in Brazil. In: Straskraba, M.; Tundisi, J. G. \& Duncan, A. eds. Comparative reservoir limnology and water management. Netherland Kluwer Academic. p.25-55. 